\title{
DUODENO-PANCREATECTOMIA CEFÁLICA NO TRATAMENTO DE ÚLCERA DUODENAL SANGRANTE NO TRANSPLANTE DE PÂNCREAS
}

\author{
Cephalic duodenopacreatectomy in the treatment of a bleeding duodenal ulcer \\ in pancreas transplants
}

\begin{abstract}
Izadora Santos Simões Pires', Rodrigo Morais de Siqueira', Leonardo V. Kroth², Marcelo Hartmann², Manlio Falavigna², Moacir Alexandre Traessel/2, Eduardo Franco Carvalhal'2, Domingos d'Avila, ${ }^{1,2}$, David Saitovitch ${ }^{1,2}$, Salvador Gullo Neto²
\end{abstract}

\begin{abstract}
RESUMO
O transplante de pâncreas constitui atualmente a única terapêutica conhecida que restabelece um estado fisiológico do metabolismo glicêmico no paciente diabético. Sua indicação mais prevalente é em associação ao transplante renal em portadores de Diabetes Mellitus tipo 1 (DM1) e nefropatia que estão sob tratamento dialítico ou na iminência dessa terapêutica. Entre as complicações do transplante simultâneo de pâncreas e rim (TxSPR), destacam-se as cirúrgicas, em especial aquelas decorrentes da derivação exócrina vesical, com elevada morbimortalidade. Neste relato, apresentamos o caso clínico de um paciente masculino, 31 anos, branco com DM1 desde os 12 anos e em hemodiálise há dois anos, que foi submetido ao TxSPR. Após, desenvolveu hematúria franca por úlcera duodenal sangrante do enxerto, sem etiologia definida. O emprego da técnica de pancreatocistostomia do pâncreas segmentar para drenagem da secreção exócrina permitiu a manutenção do enxerto e o estado euglicêmico do paciente, livre de insulina exógena.
\end{abstract}

Descritores: Transplante de Pâncreas, Úlcera Duodenal do Enxerto, Diabetes Mellitus, Sangramento

\footnotetext{
Instituições:

1 Faculdade de Medicina da Pontifícia Universidade Católica do Rio Grande do Sul, Porto Alegre, Rio Grande do Sul, Brasil.

2 Serviço de Transplantes do Hospital São Lucas da Pontifícia Universidade Católica do Rio Grande do Sul, Porto Alegre, Rio Grande do Sul, Brasil.
}

Correspondência:

Salvador Gullo Neto

Serviço de Transplantes, Hospital São Lucas da PUCRS

Avenida Ipiranga 6.690 - CEP 90610-000 - Porto Alegre/RS

Tel/Fax: (51) 3336-7700

Email: sgulloneto@yahoo.com

Recebido em: 14.10 .2009

Aceito em: 21.10.2009

\section{INTRODUÇÃo}

O transplante de pâncreas é o único tratamento disponível capaz de restabelecer um estado fisiológico no metabolismo glicêmico de paciente diabético, tornando-o livre de insulina endógena. ${ }^{1}$ A melhor opção terapêutica para pacientes com DM1 e insuficiência renal crônica em estágio final é o TxSPR, com melhora significativa da qualidade de vida. ${ }^{2}$

Entre as complicações do TxSPR, destacam-se as cirúrgicas, em especial aquelas decorrentes da derivação exócrina vesical, com elevada morbimortalidade. ${ }^{3}$ As mais freqüentes são infecções do trato urinário de repetição, uretrite, hematúria, acidose metabólica e desidratação. ${ }^{4}$

Neste relato, apresentamos o caso clínico de um paciente submetido a TxSPR que desenvolveu hematúria franca por úlcera duodenal sangrante do enxerto, sem etiologia definida, tratado por duodenopancreatectomia cefálica, preservando a porção corpo-caudal do pâncreas e com derivação vesical por pancreatocistostomia.

\section{RELATO DE CASO}

Paciente masculino, 31 anos, branco, com DM1 desde os 12 anos e em hemodiálise por dois anos foi submetido ao TxSPR há dois anos. A imunossupressão utilizada foi com tacrolimus, micofenolato mofetil 
e prednisona, sem indução. As funções renal e pancreática foram restauradas progressivamente depois do transplante, estabelecendo euglicemia e níveis normais de creatinina. Após o transplante teve diagnostico de infecção por citomegalovirus (CMV), tratada com Glanciclovir endovenoso. Um ano após o transplante, o paciente apresentou quadro de dor no baixo ventre associado à disúria intensa e hematúria. Naquele momento, o paciente apresentava lipase sérica $137 \mathrm{U} / \mathrm{L}$, amilase sérico $91 \mathrm{U} / \mathrm{L}$, creatinina $1,3 \mathrm{mg} / \mathrm{dL}$ e amilasúria 28696 U/L e 24 horas depois, $17501 \mathrm{U} / \mathrm{L}$, tais exames laboratoriais não eram sugestivos de rejeição do enxerto. Foi realizada cistoscopia, na qual foi diagnosticada úlcera duodenal do enxerto. No mesmo procedimento, coletou-se material para biópsia e realizou-se hemostasia da úlcera por eletrocoagulação. Após dois dias, evoluiu para choque não responsivo a volume. Foi submetido à laparotomia exploradora, tendo sido diagnosticada úlcera duodenal do enxerto com sangramento ativo e obstrução vesical por coágulos. Realizou-se ulcerorrafia para contenção do sangramento, preservando o enxerto pancreático. Dias após, reiniciou com dor hipogástrica e hematúria. Apresentou dois episódios de síncope com retenção urinária e hematúria franca. Durante o exame, a pressão arterial estava baixa, a freqüência cardíaca era de 122 bpm e apresentava palpitações, o globo vesical era palpável e doloroso ao toque. Depois de corrigida a volemia, o paciente foi encaminhado para cirurgia de duodenopancreatectomia cefálica, com preservação do corpo e cauda do pâncreas com derivação da secreção exócrina pancreática através de pancreaticocistostomia (Figura 1). O exame da biópsia apresentou tecido pancreático com fibrose e inflamação crônica, ausência de mucosa duodenal e pesquisa de inclusões virais, fungos e BAAR, negativa. $\mathrm{O}$ anátomo-patológico da peça cirúrgica diagnosticou úlcera com inflamação inespecífica e fibrose, e pâncreas com estrutura histológica conservada. A opção por preservar o enxerto foi tomada, pois a complicação se dava exclusivamente na cabeça do pâncreas e a função do enxerto era normal. Essa forma de reconstrução da drenagem exócrina pancreática, descrita por Sollinger et $\mathrm{al}^{5}$ para realizar transplante segmentar de pâncreas, em 1982 foi útil no resgate do enxerto e preservou o estado euglicêmico do paciente.

\section{DISCUSSÃO}

O transplante de pâncreas constitui a única terapêutica conhecida capaz de controlar variações glicêmicas, normalizar os níveis de hemoglobina glicosilada e manter condição euglicêmica e de independência da insulina exógena em portadores de DM1. ${ }^{6}$ Sua indicação mais prevalente é em associação ao transplante renal em portadores de DM1 e estágio final de insuficiência renal. ${ }^{7}$

Desde o primeiro TxSPR, realizado em 1966 por Willian Kelly e Richard Lillehei, ${ }^{8}$ ocorreram várias mudanças nesse procedimento na tentativa de aprimorar a técnica, tendo em vista os resultados iniciais não satisfatórios. ${ }^{9}$ Em 1980, o Registro Internacional de Transplante de Pâncreas mostrava índice de sobrevivência do enxerto pancreático de $21 \%$ e dos pacientes de $67 \%$ no primeiro ano. Consequentemente, a comunidade médica tornou-se relutante ao realizar o procedimento. ${ }^{10}$ Naquele mesmo ano, Groth et al. utilizaram drenagem entérica em Y de Roux, em pacientes que receberam o enxerto pancreático. ${ }^{11}$

Várias técnicas foram utilizadas, mas o maior avanço ocorreu em 1982, quando Sollinger et al. introduziram a drenagem vesical de secreção exócrina. ${ }^{5}$ Tal técnica foi modificada por Nghiem e Corry. ${ }^{12}$ Posteriormente, desde o final da década de 90 até os dias de hoje, o TxSPR passou por novas tendências, não só em relação à técnica cirúrgica, mas também quanto aos cuidados clínicos..$^{13}$ Atualmente, segundo o Registro Internacional de Transplante de Pâncreas, a sobrevida do enxerto pancreático está em torno de $83 \%$, e a sobrevida dos pacientes em torno de $95 \%$ para TxSPR no primeiro ano póstransplante. ${ }^{14}$

A drenagem vesical da secreção exócrina pode ser associada a complicações como: hematúria, cistites, uretrites, acidose metabólica, desidratação, fístulas urinárias, infecções urinárias e pancreatite do refluxo. ${ }^{15,16}$ Uma causa pouco comum de hematúria no TxSPR, é o sangramento de úlcera duodenal do enxerto, aqui relatado. Ela é geralmente auto-limitada, mas pode levar à perda do enxerto. Em alguns casos, a úlcera duodenal do enxerto é causada por infecção por CMV que, quando identificada, deve ser imediatamente tratada. ${ }^{17}$ A duodeno-pancreatectomia cefálica com preservação do corpo e cauda do pâncreas e com derivação da drenagem da secreção exócrina pancreática para a bexiga por pancreatocistostomia, descrita primeiramente para o transplante de pâncreas segmentar, ${ }^{5}$ mostrou-se uma alternativa eficaz para salvar enxerto de pâncreas com úlcera duodenal sangrante.

\section{CONCLUSÃO}

Concluímos que a técnica da duodenopancreatectomia cefálica associada à pancreatocistostomia é uma alternativa possível para o controle da hemorragia de úlcera duodenal do enxerto pancreático, com a vantagem de preservar a função do enxerto.

\section{ABSTRACT}

Pancreas transplantation is currently the only known therapy which restores glycemic metabolism in type 1 diabetic patients. The most prevalent indication is in association with kidney transplantation in patients with type 1 Diabetes Mellitus (DM1) and nephropathy, who are under dialytic treatment. It can be detached among the complications of simultaneous pancreas and kidney transplantation (SPK): surgical re-interventions, especially those resulting from complications of bladder exocrine pancreatic drainage, with considerable morbidity and mortality. In this report, we present a clinical case of a 31 years-old Caucasian male patient with DM1 since he was 12 years-old, and on hemodialysis for two years, until he was submitted to a SPK two years ago. He then developed massive hematuria due to a bleeding duodenal graft ulcer. The use of the segmental pancreatic technique with pancreaticocystostomy for exocrine pancreatic drainage allowed the maintenance of the graft and the euglycemic state of the patient free of exogenous insulin.

Keywords: Pancreas Transplantation, Duodenal Graft Ulcer, Diabetes Mellitus, Bleeding 


\section{REFERÊNCIAS}

1. Ojo AO, Meier-Kriesche H, Hanson JA, et al. The impact of simultaneous pancreaskidney transplantation on long-term patient survival. Transplantation. 2001 Jan 15;71(1):82-90

2. Ziaja J, Bozek-Pajak D, Kowalik A, et al. Impact of pancreas transplantation on the quality of life of diabetic renal transplant recipients. Transplantation proceedings. 2009 Oct;41(8):3156-8.

3. Michalak G, Kwiatkowski A, Czerwinski J, et al. Surgical complications of simultaneous pancreas-kidney transplantation: a 16-year-experience at one center Transplantation Proceedings. 2005 Oct;37(8):3555-7.

4. Medina Polo J, Morales JM, Blanco M, et al. Urological complications after simultaneous pancreas-kidney transplantation. Transplantation Proceedings. 2009 Jul-Aug;41(6):2457-9.

5. Cook K, Sollinger HW, Warner T, et al. Pancreaticocystostomy: an alternative method for exocrine drainage of segmental pancreatic allografts. Transplantation. 1983,35(6):634-636

6. Stratta RJ, Taylor RJ, Gill IS. Pancreas transplantation: a Managed Cure Approach to Diabetes. Curr Probl Surg. 1996 Sep;33 (9):709-808.

7. Decker E, Coimbra C, Weekers L, et al. A retrospective monocenter review of simultaneous pancreas-kidney transplantation. Transplantation Proceedings. 2009 Oct;41(8):3389-92.

8. Kelly WD, Lillehei RC, Merkel FK, et al. Allotransplantation of the pancreas and duodenum along with the kidney in diabetic nephropathy. Surgery. 1967;61(6):827-837.
9. Sutherland DE, Gruessner RW, Dunn DL, et al. Lessons learned from more than 1,000 pancreas transplants at a single institution. Annals of Surgery. 2001 Apr;233(4):463-501.

10. Sutherland DE. International human pancreas and islet transplant registry. Transplantation Proceedings. 1980 Dec;12(4 Suppl 2):229-36.

11. Groth CG, Lundgren G, Ostman J, et al. Experience with nine segmental pancreatic transplantations in preuremic diabetic patients in Stockholm. Transplantation Proceedings.1980;12(4 Suppl 2):68-71.

12. Nghiem DD, Corry RJ. Technique of simultaneous renal pancreatoduodenal transplantation with urinary drainage of pancreatic secretion. American Journal of Surgery.1987;153(4):405-406.

13. Sollinger HW, Odorico JS, Knechtle SJ, et al. Experience with 500 simultaneous pancreas-kidney transplants. Annals of Surgery. 1998;228(3):284-96.

14. Gruessner AC, Sutherland DE. Analysis of United States (US) and non-US pancreas transplants reported to the United network for organ sharing (UNOS) and the international pancreas transplant registry (IPTR) as of October 2001. Clinical Transplants. 2001;41-72

15. Ciancio G, Burke GW, Nery JR, et al. Urethritis/dysuria after simultaneous pancreaskidney transplantation. Clinical Transplantation. 1996,10(1 Pt 1):67-70.

16. Ciancio G, Burke GW, Roth D, et al. Reflux pancreatitis after simultaneous pancreaskidney transplantation treated by alpha 1 blocker. Transplantation. 1995,60(7):760-1

17. Gary W, Barone, M.D., John W. Webb, et al. the enteric drained pancreas transplant: another potential source of gastrointestinal bleeding. The American Journal of Gastroenterology. Aug;93(8):1369-71 